\title{
Entrepreneurship and welfare
}

\author{
Jagannadha Pawan Tamvada
}

Accepted: 15 March 2009/Published online: 6 May 2009

(C) The Author(s) 2009. This article is published with open access at Springerlink.com

\begin{abstract}
Here, I examine returns to entrepreneurship using a standard measure of welfare, the percapita consumption expenditure. This analysis, using quantile regressions, reveals the existence of a welfare hierarchy in occupations. The results suggest that, across the welfare distribution, entrepreneurs who employ others have the highest returns in terms of consumption, while those entrepreneurs who work for themselves, that is, self-employed individuals, have slightly lower returns than the salaried employees. However, self-employment entails higher returns than casual labor and a relative escape from poverty.
\end{abstract}

Keywords Developing countries .

Entrepreneurship · Quantile regressions ·

Self-employment · Welfare

Discussions with David Audretsch, Stephan Klasen, Wim Naude, and Christina Schneider have been very useful in the preparation of this manuscript. I am grateful to an anonymous referee, participants of the IZA-World Bank Conference on Employment and Development, the UNU-WIDER Workshop on Entrepreneurship and Economic Development, and faculty seminars at the University of Göttingen and Max Planck Institute for helpful comments.

J. P. Tamvada ( ()

Entrepreneurship, Growth and Public Policy Group, Max

Planck Institute of Economics, Kahlaische Strasse 10,

07745 Jena, Germany

e-mail: tamvada@econ.mpg.de
JEL Classifications $\quad$ J24 $\cdot$ J43 $\cdot$ J44 $\cdot$ L26

\section{Introduction}

There is a rich literature providing insights into the determinants of entrepreneurship and its economic returns. ${ }^{1}$ According to the expected utility theory, individuals choose self-employment when they expect higher returns from doing so relative to wage-employment (Rees and Shaw 1986). In contrast, according to the non-pecuniary benefits theory, people select into entrepreneurship, even if the expected returns are lower, in search of non-pecuniary benefits, such as being their own boss (Hamilton 2000). However, entrepreneurs are not a homogenous group of individuals, and the type of entrepreneurship engaged in may have a significant effect on the returns. $^{2}$

To date, there has been little research into the nature of entrepreneurship and its economic returns in developing countries. The purpose of this paper is to examine the welfare effects of different types of

\footnotetext{
1 See Parker (2004) for a synthesis of this literature.

${ }^{2}$ For example, while some entrepreneurs employ others, the rest are solely self-employed individuals.
} 
entrepreneurship in the context of a developing country. Using a direct measure of welfare, percapita consumption expenditure, and quantile regressions, this study examines the returns to individuals' occupational choice across the welfare distribution. ${ }^{3}$ The results suggest that, across the welfare distribution, entrepreneurs who employ others have the highest returns in terms of consumption, while those entrepreneurs who work for themselves, that is, selfemployed individuals, have slightly lower returns than the salaried employees. However, self-employment entails higher returns than casual labor and a relative escape from poverty.

The structure of the paper is as follows. Section 2 provides an overview of the theoretical background on occupational choice and welfare and sets out the hypotheses. The third section discusses the methodology employed in this paper to examine the returns to occupations across the welfare distribution, the quantile regressions. Data and descriptive statistics are presented in the fourth section, and the fifth section contains a discussion of the empirical analysis linking occupation and welfare. The paper concludes with a summary of the main findings linking occupation and welfare.

\section{Theoretical background}

A key observation of many studies, including that of Banerjee and Neuman (1993) and, more recently, that of Dabla-Norris et al. (2008), is the inherent hierarchy of occupational choice according to which the most productive individuals become entrepreneurs, the next best choose self-employment, and the rest become workers or subsistence workers. DablaNorris et al. (2008) propose that at equilibrium, the lowest productivity individuals are workers, individuals with intermediate productivity are informal entrepreneurs, and those who are most productive

\footnotetext{
${ }^{3}$ Most studies use income measures to examine the returns of occupations (Hamilton 2000). In this paper, we use consumption measures. Income is usually highly correlated with consumption (Browning and Lusardi 1996). An analysis of the consumption patterns itself has the advantage that variation is not so high as in income data. However, as people with higher incomes are likely to have greater savings, an analysis of the consumption patterns for welfare comparisons may make their returns appear flattened to some extent.
}

are formal sector entrepreneurs. These theoretical insights have yet to be empirically validated. The possibility of self-employment being worse off in the hierarchy relative to wage workers, as is traditionally assumed to be the case in less developed countries (Ranis and Fei 1961; Harris and Todaro 1970), or at least equal in returns, would contest the applicability of these theories to less developed countries (LDCs). The literature on LDCs traditionally identifies selfemployment as a distressed residual of people rationed out of jobs in the formal sector, although more recent literature on the nature of the labor market in developing countries is not monolithic on this point. Some scholars believe that the informal sector in LDCs consists of voluntarily self-selected competitive workers as well as disadvantaged individuals (Gindling 1991; Magnac 1991; Cunningham and Maloney 2001; Maloney 2004; Fields 2005; Günther and Launov 2006). ${ }^{4}$

Occupational choice is generally modeled as a utility-maximizing decision of individuals (Lucas 1978; Kihlstrom and Laffont 1979). ${ }^{5}$ While many models in the economics of entrepreneurship assume that individuals become self-employed as they expect higher returns relative to wage employment (Rees and Shaw 1986; Blau 1987; Parker 1996), the labor and development literature suggests that in the LDC context, people are forced into self-employment in the absence of viable economic opportunities.

However, empirical studies, such as that of Hamilton (2000), that focus on developed countries suggest that entrepreneurs may trade lower earnings for the non-pecuniary benefits of business ownership. ${ }^{6}$ Evans and Leighton (1989) suggest that individuals who prefer greater autonomy are more

\footnotetext{
${ }^{4}$ Pratap and Quintin (2006) argue that there is no evidence of market segmentation in the labor markets of developing countries.

5 There are two main methods to model the returns of occupational choice. The first is to estimate a mincer type wage equation for each occupation; the second is the structural probit method that estimates the reduced form probit and determines the wages corrected for selection. The sign of mill's ratio indicates the nature of selection. The predicted earnings differential are used to re-estimate the probit equation to predict the self-employment choice as a function of expected utility (Rees and Shaw 1986).

${ }^{6}$ Hamilton (2000) finds no evidence of the earnings differential being a result of the selection of low-ability employees into self-employment. Further, he argues that, for most
} 
likely to be entrepreneurs. Blanchflower and Oswald (1998) show that business owners have greater job satisfaction than paid-employees. According to Boháček (2006), as successful firms grow over time, individuals may enter self-employment even if the returns are lower.

Thus, two main theories of returns to selfemployment choice have emerged. First, the expected utility view claims that individuals choose selfemployment when they expect higher returns in self-employment relative to wage-employment. This theory also explains the traditional low-productivity view of self-employment, which suggests that individuals are compelled into self-employment in the absence of viable economic alternatives. Second, the non-pecuniary benefits view argues that individuals select into entrepreneurship even when the returns are lower, for non-pecuniary benefits, such as being one's boss.

I hypothesize that, given the occupational structure of individuals in an economy, the returns to occupations depend on the relative positioning of individuals in the welfare distribution. Self-employed individuals at the lower end of the distribution fundamentally differ from the self-employed individuals in the upper end of the distribution. This is also true for salaried employees. Occupations and their economic returns are characterized by a heterogeneity that is not discernable in studies that examine this relationship solely at the mean. By examining the returns to occupations across the welfare distribution, this paper sets out a novel approach to studying the relative returns to occupations.

A number of other factors that have been found to influence the per-capita consumption of the households are examined in this study. Dreze and Srinivasan (1997), using an earlier survey of India's National Sample Survey Organization (NSSO), find that households that are female headed are more likely to be poor. Jenkins (2000) finds that changes in labor earnings from persons other than the household head, changes in non-labor income, changes in the earnings of the household head, and household composition are important determinants of the poverty dynamics. Miles (1997) finds

Footnote 6 continued entrepreneurs, self-employment offers significant nonpecuniary benefits, such as being one's own boss. that uncertainty, education, and location matter. Using both durable and non-durable goods in the welfare measure, Glewwe (1991) finds high returns to education in urban areas compared to rural areas in Côte d'Ivory. Nelson (1988) shows the existence of economies of scale in all adult households. Such economies of scale are found to be more important in the consumption of shelter and less so in the consumption of clothing and transportation. ${ }^{7}$ Furthermore, a vast literature is concerned with equivalence scales in the measurements of welfare for comparisons across households. Households with the same income but different structures, in terms of the number of children and old people, are likely to have different consumption patterns. For example, Lanjouw and Ravallion (1995, pp. 1431-1432) suggest that the relationship between poverty and household size depends on the weight attached to child and adult welfare. ${ }^{8}$ Hence, household demographic structure is controlled in the analysis reported here. In the Indian context, Dreze and Srinivasan (1997) find that the poverty head-count ratio is very robust to alternate equivalent scales. I also test the robustness of the results using adult equivalent scales. ${ }^{9}$

\footnotetext{
$\overline{7}$ Economies of scale have a range of $0-1$, with 1 indicating no economies of scale; the measure of welfare considering the economies of scale is equal to per-capita income of the household in this case. Here, however, I use the standard measure of welfare, per-capita expenditure on consumption. One of the reasons for using the standard measure in the analysis is that although all nonagricultural households are used in the beginning, the rest of the analysis is restricted to those households where the sole economically activate member is the household head. Thus, it is plausible to assume economies of scale close to 1 in such households.

8 They find evidence against the conventional view that household size is negatively correlated to welfare when the Rothbarth method based on non-food spending is used as a measure of welfare while a measure based on child stunting indicates that larger households tend to be poor. Browning (1992) notes that although children may be endogenous to whatever we are interested in modeling, this can be circumvented by assuming that fertility is exogenous. See Browning and Crossley (2001) for recent developments in the life cycle model of consumption. More recent approaches to measuring poverty using perceptions of consumption adequacy are addressed in Pradhan and Ravallion (2000).

9 The results are not reported in the paper but are available on request from the author.
} 


\section{Methodology}

For testing the hypothesis of heterogenous returns of occupation across the welfare distribution, I employ quantile regressions (see Koenker and Hallock 2001, and references therein). The superstar model of Rosen (1981) suggests that a comparison of the mean earnings of workers in self-employed sector and in wage sector would be highly influenced by a few entrepreneurial superstars. Thus, mean earnings do not really characterize the returns of the majority of self-employed individuals. The greatest advantage of using quantile regressions is their ability to show snapshots of relationships across different quantiles of the distribution and not only at the mean.

\section{Data}

The data used for the analysis originate from the 60th round employment-unemployment survey of the National Sample Survey Organization (NSSO) of India. I only consider those households where the household heads are reported to be self-employed (includes own account workers and employers), salaried employees, casual laborers, and unemployed. The sample is restricted to those who are older than 15 years but younger than 70 years. I then consider only those households who work in the nonagriculture sectors. The final sample consists of 26,485 households, of which only the household head is economically active in 13,782 households.

Table 1 reports summary statistics of the database. The first two data columns report the mean and standard deviation of the variables when the entire database is considered. The third and fourth column report the descriptive statistics when the database is restricted to households that have the household head as the sole economically active member. As the descriptive data on monthly per-capita consumption expenditure (MPCE) in columns 1 and 3 show, employers have the highest average consumption rate. The self-employed individuals have an consumption rate that is lower than that of salaried employees but higher than the consumption rate of the casual laborers.

Figure 1 shows that kernel density plots of log percapita consumption of households with heads working as self-employed, salaried employees, employers, and laborers. While the distribution plots of salaried employees and employers are to the right of the selfemployed, the density of the laborers is centered to their left. The plots also show that the inequality observed in the employer group is substantially higher than that in the other groups.

\section{Empirical results}

\subsection{Entrepreneurship and welfare}

\subsubsection{Household level analysis}

The empirical strategy is to estimate simultaneous quantile regressions, using the $\log$ of per-capita consumption of the household as dependent variable. ${ }^{10}$ As Browning and Lusardi (1996, p. 1801) note 'although consumption changes are uncorrelated with anticipated income changes, the actual path of consumption may follow quite closely the actual path of income if the latter displays some persistence.' Hence, the consumption and income paths are assumed to be correlated.

The occupations of the members of the household enter the regression as independent variables. A series of controls that are found to influence the consumption of the household by earlier studies are introduced in the estimation. In particular, personal characteristics of the household head, demographics of the household, including the proportion of children, adults, and old persons, educational background of the members, urban location, and land possessed are introduced as control variables. ${ }^{11}$ State level dummies are also included to control for regional effects.

\footnotetext{
$\overline{10}$ Wodon (2000) also uses per-capita consumption. Many alternate strategies to construct welfare measures that are comparable across households exist. For instance, Lazear and Michael (1980) develop a technique that converts families of different structures into single person equivalents. Also see Muellbauer (1974) and Deaton and Muellbauer (1980, 1986) for a theory of equivalence scales. The identification of correct equivalent scales is still an unresolved issue (Deaton and Paxson 1995).

${ }^{11}$ Land variables proxy the wealth of the household. Wodon (2000) suggests that the land possessed by a household is also a determinant of the welfare. I also check for the robustness of the results with the land variables excluded from the analysis. Given that only nonagricultural households are included in the data set, the problem of endogeneity of the land variables is not an issue.
} 
Table 1 Summary statistics

The first two columns report the mean and standard deviation of variables in the database. The third and fourth columns report the mean and standard deviation when the sample is restricted to those households where the household head is the only economically active individual

\begin{tabular}{|c|c|c|c|c|}
\hline \multirow[t]{2}{*}{ Variables } & \multicolumn{2}{|c|}{ Households } & \multicolumn{2}{|c|}{ Household heads } \\
\hline & Mean & $\begin{array}{l}\text { Standard } \\
\text { deviation }\end{array}$ & Mean & $\begin{array}{l}\text { Standard } \\
\text { deviation }\end{array}$ \\
\hline \multicolumn{5}{|l|}{ Consumption } \\
\hline Log(MPCE-all) & 6.63 & 0.63 & 6.71 & 0.64 \\
\hline Log(MPCE-employers) & 7.27 & 0.58 & 7.29 & 0.59 \\
\hline Log(MPCE-salaried) & 6.84 & 0.61 & 6.92 & 0.61 \\
\hline Log(MPCE-self-employed) & 6.52 & 0.59 & 6.59 & 0.60 \\
\hline Log(MPCE-laborers) & 6.25 & 0.47 & 6.28 & 0.50 \\
\hline \multicolumn{5}{|l|}{ Occupation } \\
\hline Self-employed & 0.40 & 0.49 & 0.37 & 0.48 \\
\hline Employers & 0.01 & 0.12 & 0.02 & 0.12 \\
\hline Salaried employees & 0.42 & 0.49 & 0.47 & 0.50 \\
\hline Laborer & 0.16 & 0.36 & 0.15 & 0.35 \\
\hline Unemployed & 0.01 & 0.09 & 0.01 & 0.08 \\
\hline \multicolumn{5}{|l|}{ Personal characteristics } \\
\hline Age & 41.96 & 10.71 & 38.36 & 9.69 \\
\hline Female & 0.05 & 0.22 & 0.05 & 0.22 \\
\hline Married & 0.90 & 0.30 & 0.89 & 0.31 \\
\hline Divorce/Widow & 0.05 & 0.22 & 0.04 & 0.20 \\
\hline \multicolumn{5}{|l|}{ Education } \\
\hline Informal education & 0.09 & 0.28 & 0.07 & 0.26 \\
\hline Primary school & 0.32 & 0.47 & 0.31 & 0.46 \\
\hline High school & 0.26 & 0.44 & 0.29 & 0.45 \\
\hline University education & 0.16 & 0.37 & 0.20 & 0.40 \\
\hline Technical degree or diploma & 0.07 & 0.25 & 0.08 & 0.27 \\
\hline \multicolumn{5}{|l|}{ Household variables } \\
\hline Proportion children $(<5$ years) & 0.10 & 0.15 & 0.12 & 0.17 \\
\hline Proportion children (6-10 years) & 0.09 & 0.14 & 0.11 & 0.16 \\
\hline Proportion children (11-15 years) & 0.09 & 0.14 & 0.10 & 0.15 \\
\hline Proportion females (15-60 years) & 0.32 & 0.17 & 0.30 & 0.19 \\
\hline Proportion males (15-60 years) & 0.39 & 0.22 & 0.37 & 0.25 \\
\hline Proportion old ( $>60$ years) & 0.01 & 0.05 & 0.01 & 0.05 \\
\hline Urban & 0.59 & 0.49 & 0.62 & 0.49 \\
\hline Land Code 1 & 0.35 & 0.48 & 0.40 & 0.49 \\
\hline Land Code 2 & 0.53 & 0.50 & 0.52 & 0.50 \\
\hline Land Code 3 & 0.09 & 0.29 & 0.07 & 0.25 \\
\hline Land Code 4 & 0.02 & 0.15 & 0.01 & 0.12 \\
\hline Household Size & 4.80 & 2.31 & 4.00 & 1.76 \\
\hline Manufacturing & 0.22 & 0.41 & 0.21 & 0.41 \\
\hline Trade & 0.21 & 0.41 & 0.20 & 0.40 \\
\hline Service & 0.26 & 0.44 & 0.27 & 0.45 \\
\hline Public & 0.18 & 0.38 & 0.19 & 0.39 \\
\hline$n$ & 26591 & & 14000 & \\
\hline
\end{tabular}



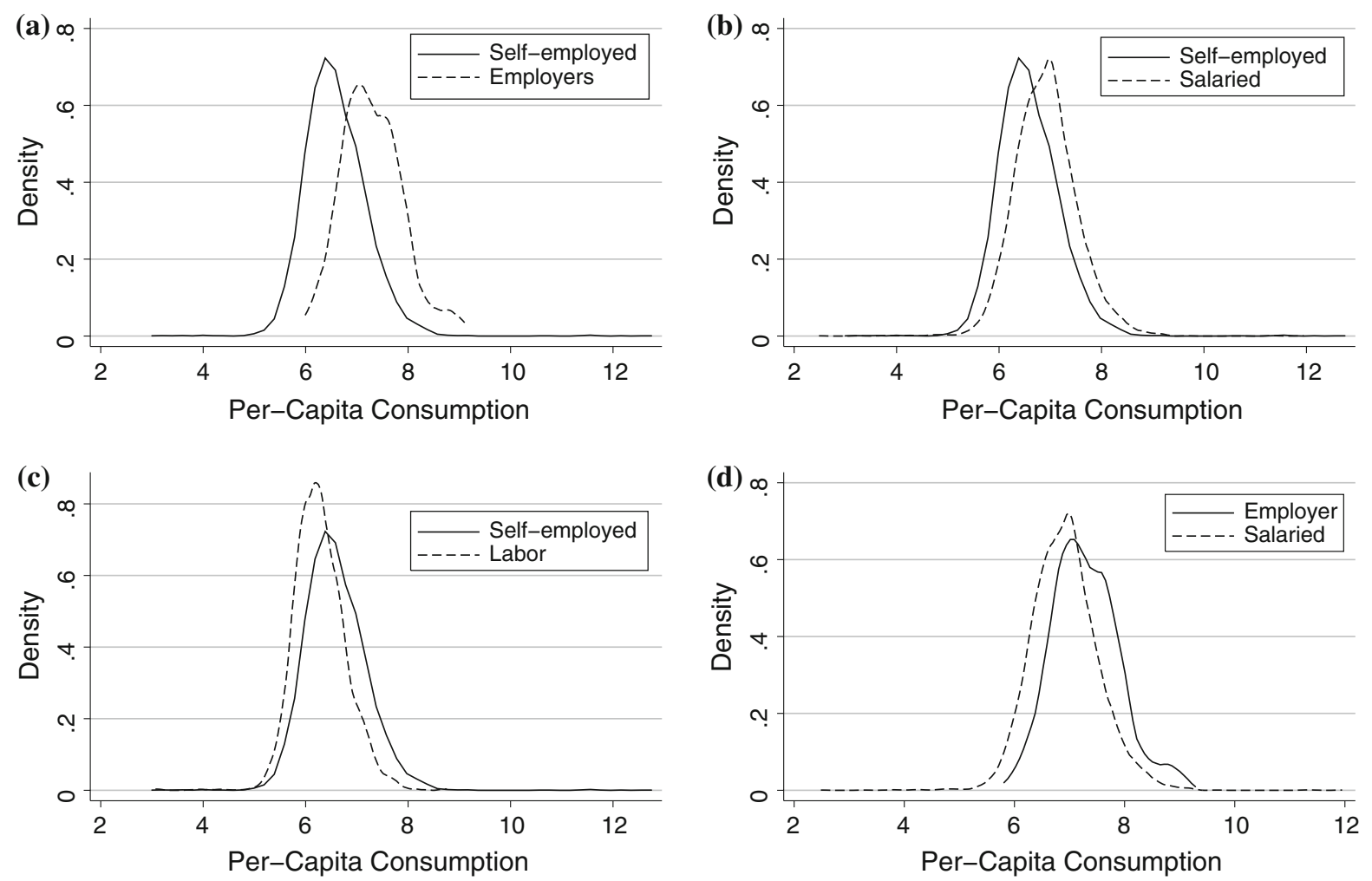

Fig. 1 Consumption and occupation (un-normalized)

The results presented in Table 2 suggest that the entrepreneurship has a distinct relationship with welfare. As mentioned earlier, people in the database belong to one of the five primary occupations-they are either employers, self-employed, salaried employees, casual laborers, or unemployed. In this estimation, the category that is left out of the occupation variables is the proportion of economically active individuals in a household who are self-employed. As the positive coefficients suggest, households that have a higher proportion of employers and those that a higher proportion of salaried employees have higher per-capita consumption levels than self-employed households. However, households that have a higher proportion of casual laborers and unemployed people have lower welfare levels than self-employed households. This result suggests the existence of a welfare hierarchy that is determined by the occupational choices of the members of the household.

As the estimates in Table 2 suggest, employers are increasingly better off at higher quantiles than selfemployed workers. Salaried employees who are in the middle of the distribution show the greatest

difference from the self-employed individuals compared to those at the extreme quantiles. At higher quantiles, casual laborers are increasingly worse off than the self-employed individuals, and a similar phenomenon is observed for the unemployed. ${ }^{12}$

The coefficients of control variables are in accordance with what might be expected. Households with older household heads are more likely to have higher consumption rates, and female-headed households are poorer across quantiles. Female-headed households are the most worse off at the lowest quantile of the distribution. Households with a higher proportion of educated individuals have higher consumption rates, and the returns are increasing along the quantiles as well as along higher levels of education. The proportion of children $<15$ years old in the household has a significant negative effect at the lowest two quantiles, but this vanishes at higher quantiles. However, the proportion of old people in a household significantly increases the per-capita consumption

\footnotetext{
$\overline{12}$ However, the unemployed variable slightly moves upward at the highest quantile but remains significantly negative.
} 
Table 2 Households, occupation, and consumption

\begin{tabular}{|c|c|c|c|c|c|}
\hline \multicolumn{6}{|c|}{ Estimates of simultaneous quantile regression } \\
\hline Independent variable & q10 & $\mathrm{q} 25$ & $\mathrm{q} 50$ & $\mathrm{q} 75$ & q90 \\
\hline \multicolumn{6}{|l|}{ Occupation } \\
\hline Proportion employers & $\begin{array}{l}0.336 * * * \\
(0.038)\end{array}$ & $\begin{array}{l}0.342 * * * \\
(0.047)\end{array}$ & $\begin{array}{l}0.405 * * * \\
(0.039)\end{array}$ & $\begin{array}{l}0.454 * * * \\
(0.035)\end{array}$ & $\begin{array}{l}0.461 * * * \\
(0.045)\end{array}$ \\
\hline Proportion salaried & $\begin{array}{l}0.0816^{* * * *} \\
(0.011)\end{array}$ & $\begin{array}{l}0.0945^{* * * *} \\
0.0081)\end{array}$ & $\begin{array}{l}0.0996 * * * \\
0.0077)\end{array}$ & $\begin{array}{l}0.0841^{* * * *} \\
0.0069)\end{array}$ & $\begin{array}{l}0.0778^{* * *} \\
(0.013)\end{array}$ \\
\hline Proportion laborers & $\begin{array}{l}-0.148^{* * * *} \\
(0.012)\end{array}$ & $\begin{array}{l}-0.143 * * * \\
(0.011)\end{array}$ & $\begin{array}{l}-0.158 * * * \\
(0.010)\end{array}$ & $\begin{array}{l}-0.172 * * * \\
(0.012)\end{array}$ & $\begin{array}{l}-0.184 * * * \\
(0.016)\end{array}$ \\
\hline Proportion unemployed & $\begin{array}{l}-0.192 * * * \\
(0.032)\end{array}$ & $\begin{array}{l}-0.187 * * * \\
(0.017)\end{array}$ & $\begin{array}{l}-0.208^{* * *} \\
(0.027)\end{array}$ & $\begin{array}{l}-0.242 * * * \\
(0.020)\end{array}$ & $\begin{array}{l}-0.182^{* * * *} \\
(0.043)\end{array}$ \\
\hline \multicolumn{6}{|l|}{ Head's characteristics } \\
\hline Age & $\begin{array}{l}0.0164 * * * \\
(0.0038)\end{array}$ & $\begin{array}{l}0.0162 * * * \\
(0.0019)\end{array}$ & $\begin{array}{l}0.0184 * * * \\
(0.0016)\end{array}$ & $\begin{array}{l}0.0204 * * * \\
(0.0026)\end{array}$ & $\begin{array}{l}0.0163 * * * \\
(0.0050)\end{array}$ \\
\hline Age square & $\begin{array}{l}-0.0163 * * * \\
(0.0042)\end{array}$ & $\begin{array}{l}-0.0156^{* * *} \\
(0.0022)\end{array}$ & $\begin{array}{l}-0.0174 * * * \\
(0.0018)\end{array}$ & $\begin{array}{l}-0.0193 * * * \\
(0.0032)\end{array}$ & $\begin{array}{l}-0.0146^{* *} \\
(0.0057)\end{array}$ \\
\hline Female & $\begin{array}{l}-0.0912 * * * \\
(0.025)\end{array}$ & $\begin{array}{l}-0.0896 * * * \\
(0.025)\end{array}$ & $\begin{array}{l}-0.0738 * * * \\
(0.014)\end{array}$ & $\begin{array}{l}-0.0801 * * * \\
(0.021)\end{array}$ & $\begin{array}{l}-0.0573^{* *} \\
(0.025)\end{array}$ \\
\hline Married & $\begin{array}{l}0.0516^{*} \\
(0.028)\end{array}$ & $\begin{array}{l}0.0459 * * * \\
(0.017)\end{array}$ & $\begin{array}{l}0.0495 * * * \\
(0.016)\end{array}$ & $\begin{array}{l}0.0261 \\
(0.025)\end{array}$ & $\begin{array}{l}0.00218 \\
(0.031)\end{array}$ \\
\hline Divorce/widow & $\begin{array}{l}-0.0382 \\
(0.042)\end{array}$ & $\begin{array}{l}-0.0242 \\
(0.026)\end{array}$ & $\begin{array}{l}-0.0285 \\
(0.025)\end{array}$ & $\begin{array}{l}-0.0162 \\
(0.030)\end{array}$ & $\begin{array}{l}-0.0205 \\
(0.044)\end{array}$ \\
\hline \multicolumn{6}{|l|}{ Education } \\
\hline Proportion informal education & $\begin{array}{l}0.196 * * * \\
(0.022)\end{array}$ & $\begin{array}{l}0.200 * * * \\
(0.012)\end{array}$ & $\begin{array}{l}0.220 * * * \\
(0.010)\end{array}$ & $\begin{array}{l}0.214 * * * \\
(0.017)\end{array}$ & $\begin{array}{l}0.238 * * * \\
(0.033)\end{array}$ \\
\hline Proportion primary school & $\begin{array}{l}0.343 * * * \\
(0.021)\end{array}$ & $\begin{array}{l}0.344 * * * \\
(0.014)\end{array}$ & $\begin{array}{l}0.365^{* * *} \\
(0.013)\end{array}$ & $\begin{array}{l}0.381 * * * \\
(0.017)\end{array}$ & $\begin{array}{l}0.422 * * * \\
(0.024)\end{array}$ \\
\hline Proportion high school & $\begin{array}{l}0.565^{* * * *} \\
(0.024)\end{array}$ & $\begin{array}{l}0.602 * * * \\
(0.017)\end{array}$ & $\begin{array}{l}0.661 * * * \\
(0.018)\end{array}$ & $\begin{array}{l}0.704 * * * \\
(0.019)\end{array}$ & $\begin{array}{l}0.758 * * * \\
(0.028)\end{array}$ \\
\hline Proportion university education & $\begin{array}{l}0.958 * * * \\
(0.019)\end{array}$ & $\begin{array}{l}1.072 * * * \\
(0.020)\end{array}$ & $\begin{array}{l}1.187 * * * \\
(0.020)\end{array}$ & $\begin{array}{l}1.335 * * * \\
(0.032)\end{array}$ & $\begin{array}{l}1.519 * * * \\
(0.031)\end{array}$ \\
\hline Proportion technical degree & $\begin{array}{l}0.190 * * * \\
(0.020)\end{array}$ & $\begin{array}{l}0.235 * * * \\
(0.017)\end{array}$ & $\begin{array}{l}0.253 * * * \\
(0.033)\end{array}$ & $\begin{array}{l}0.281 * * * \\
(0.038)\end{array}$ & $\begin{array}{l}0.305 * * * \\
(0.035)\end{array}$ \\
\hline \multicolumn{6}{|l|}{ Demographics } \\
\hline Proportion children $(<5$ years $)$ & $\begin{array}{l}-0.133 * * * \\
(0.025)\end{array}$ & $\begin{array}{l}-0.0732 * * * \\
(0.023)\end{array}$ & $\begin{array}{l}-0.0156 \\
(0.032)\end{array}$ & $\begin{array}{l}0.00982 \\
(0.027)\end{array}$ & $\begin{array}{l}0.0198 \\
(0.053)\end{array}$ \\
\hline Proportion children (6-10 years) & $\begin{array}{l}-0.125^{* * * *} \\
(0.036)\end{array}$ & $\begin{array}{l}-0.0638 * * \\
(0.025)\end{array}$ & $\begin{array}{l}0.0116 \\
(0.028)\end{array}$ & $\begin{array}{l}0.0301 \\
(0.037)\end{array}$ & $\begin{array}{l}0.0981 * \\
(0.052)\end{array}$ \\
\hline Proportion children (11-15 years) & $\begin{array}{l}-0.140 * * * \\
(0.035)\end{array}$ & $\begin{array}{l}-0.0941 * * * \\
(0.022)\end{array}$ & $\begin{array}{l}-0.0601 * \\
(0.032)\end{array}$ & $\begin{array}{l}-0.0500 * \\
(0.027)\end{array}$ & $\begin{array}{l}-0.0402 \\
(0.048)\end{array}$ \\
\hline Proportion females (15-60 years) & $\begin{array}{l}0.000581 \\
(0.020)\end{array}$ & $\begin{array}{l}0.0323 \\
(0.021)\end{array}$ & $\begin{array}{l}0.0442 * * \\
(0.018)\end{array}$ & $\begin{array}{l}0.0604 * * \\
(0.025)\end{array}$ & $\begin{array}{l}0.0900 * * \\
(0.039)\end{array}$ \\
\hline Proportion old ( $>60$ years) & $\begin{array}{l}0.188 * * * \\
(0.067)\end{array}$ & $\begin{array}{l}0.196 * * * \\
(0.041)\end{array}$ & $\begin{array}{l}0.212 * * * \\
(0.060)\end{array}$ & $\begin{array}{l}0.336 * * * \\
(0.082)\end{array}$ & $\begin{array}{l}0.383 * * * \\
(0.11)\end{array}$ \\
\hline
\end{tabular}


Table 2 continued

\begin{tabular}{|c|c|c|c|c|c|}
\hline \multicolumn{6}{|c|}{ Estimates of simultaneous quantile regression } \\
\hline Independent variable & q10 & $\mathrm{q} 25$ & $\mathrm{q} 50$ & $\mathrm{q} 75$ & q90 \\
\hline \multicolumn{6}{|l|}{ Household characteristics } \\
\hline Urban & $\begin{array}{l}0.232 * * * \\
(0.0078)\end{array}$ & $\begin{array}{l}0.233 * * * \\
(0.0044)\end{array}$ & $\begin{array}{l}0.258 * * * \\
(0.0065)\end{array}$ & $\begin{array}{l}0.277 * * * \\
(0.0066)\end{array}$ & $\begin{array}{l}0.281 * * * \\
(0.0100)\end{array}$ \\
\hline $0.2<$ Land $<0.4$ ha & $\begin{array}{l}0.0415 * * * \\
(0.0086)\end{array}$ & $\begin{array}{l}0.0341 * * * \\
(0.0059)\end{array}$ & $\begin{array}{l}0.0288 * * * \\
(0.0072)\end{array}$ & $\begin{array}{l}0.0230 * * \\
(0.0091)\end{array}$ & $\begin{array}{l}0.0327 * * * \\
(0.013)\end{array}$ \\
\hline $0.4<$ Land $<2$ ha & $\begin{array}{l}0.0763 * * * \\
(0.015)\end{array}$ & $\begin{array}{l}0.0594 * * * \\
(0.011)\end{array}$ & $\begin{array}{l}0.0430 * * * \\
(0.013)\end{array}$ & $\begin{array}{l}0.0439 * * * \\
(0.017)\end{array}$ & $\begin{array}{l}0.0518 * * \\
0.021)\end{array}$ \\
\hline Land $>2$ ha & $\begin{array}{l}0.127 * * * \\
(0.018)\end{array}$ & $\begin{array}{l}0.126 * * * \\
(0.022)\end{array}$ & $\begin{array}{l}0.148 * * * \\
(0.027)\end{array}$ & $\begin{array}{l}0.147 * * * \\
(0.016)\end{array}$ & $\begin{array}{l}0.173 * * * \\
(0.030)\end{array}$ \\
\hline Household size & $\begin{array}{l}-0.118 * * * \\
(0.0045)\end{array}$ & $\begin{array}{l}-0.140 * * * \\
(0.0049)\end{array}$ & $\begin{array}{l}-0.162 * * * \\
(0.0048)\end{array}$ & $\begin{array}{l}-0.184 * * * \\
(0.0080)\end{array}$ & $\begin{array}{l}-0.206 * * * \\
(0.0086)\end{array}$ \\
\hline Householdsize square & $\begin{array}{l}0.00447 * * * \\
(0.00029)\end{array}$ & $\begin{array}{l}0.00578 * * * \\
(0.00029)\end{array}$ & $\begin{array}{l}0.00686 * * * \\
(0.00032)\end{array}$ & $\begin{array}{l}0.00838 * * * \\
(0.00062)\end{array}$ & $\begin{array}{l}0.00985 * * * \\
(0.00064)\end{array}$ \\
\hline \multicolumn{6}{|l|}{ Region controls } \\
\hline \multicolumn{6}{|l|}{ North \& East states } \\
\hline Punjab & $\begin{array}{l}0.162 * * * \\
(0.013)\end{array}$ & $\begin{array}{l}0.109 * * * \\
(0.021)\end{array}$ & $\begin{array}{l}0.0714 * * * \\
(0.015)\end{array}$ & $\begin{array}{l}0.0571 * * * \\
(0.022)\end{array}$ & $\begin{array}{l}0.0433 \\
(0.037)\end{array}$ \\
\hline Delhi & $\begin{array}{l}0.184 * * * \\
(0.016)\end{array}$ & $\begin{array}{l}0.180 * * * \\
(0.024)\end{array}$ & $\begin{array}{l}0.135 * * * \\
(0.021)\end{array}$ & $\begin{array}{l}0.0970 * * * \\
(0.021)\end{array}$ & $\begin{array}{l}0.0604 * * \\
(0.030)\end{array}$ \\
\hline Rajasthan & $\begin{array}{l}0.0802 * * * \\
(0.019)\end{array}$ & $\begin{array}{l}0.0535^{* * * *} \\
(0.012)\end{array}$ & $\begin{array}{l}-0.00930 \\
(0.015)\end{array}$ & $\begin{array}{l}-0.0596 * * * \\
(0.012)\end{array}$ & $\begin{array}{l}-0.102 * * * \\
(0.036)\end{array}$ \\
\hline Uttar Pradesh & $\begin{array}{l}-0.0687 * * * \\
(0.011)\end{array}$ & $\begin{array}{l}-0.0729 * * * \\
(0.0096)\end{array}$ & $\begin{array}{l}-0.103 * * * \\
(0.0073)\end{array}$ & $\begin{array}{l}-0.130 * * * \\
(0.014)\end{array}$ & $\begin{array}{l}-0.149 * * * \\
(0.018)\end{array}$ \\
\hline Bihar & $\begin{array}{l}-0.171^{* * *} \\
(0.018)\end{array}$ & $\begin{array}{l}-0.197 * * * \\
(0.016)\end{array}$ & $\begin{array}{l}-0.257 * * * \\
(0.016)\end{array}$ & $\begin{array}{l}-0.281^{* * *} \\
(0.019)\end{array}$ & $\begin{array}{l}-0.330 * * * \\
(0.019)\end{array}$ \\
\hline Manipur & $\begin{array}{l}0.0381 \\
(0.032)\end{array}$ & $\begin{array}{l}-0.0538 * * * \\
(0.018)\end{array}$ & $\begin{array}{l}-0.126^{* * *} \\
(0.013)\end{array}$ & $\begin{array}{l}-0.195 * * * \\
(0.019)\end{array}$ & $\begin{array}{l}-0.265^{* * *} \\
(0.034)\end{array}$ \\
\hline Assam & $\begin{array}{l}-0.0702 * * * \\
(0.025)\end{array}$ & $\begin{array}{l}-0.0766^{* * * *} \\
(0.019)\end{array}$ & $\begin{array}{l}-0.111 * * * \\
(0.014)\end{array}$ & $\begin{array}{l}-0.159 * * * \\
(0.012)\end{array}$ & $\begin{array}{l}-0.221 * * * \\
(0.021)\end{array}$ \\
\hline West Bengal & $\begin{array}{l}-0.0712 * * * \\
(0.012)\end{array}$ & $\begin{array}{l}-0.0617 * * * \\
(0.013)\end{array}$ & $\begin{array}{l}-0.106^{* * *} \\
(0.0079)\end{array}$ & $\begin{array}{l}-0.132 * * * \\
(0.0080)\end{array}$ & $\begin{array}{l}-0.160 * * * \\
(0.020)\end{array}$ \\
\hline Orissa & $\begin{array}{l}-0.310^{* * *} \\
(0.020)\end{array}$ & $\begin{array}{l}-0.328 * * * \\
(0.013)\end{array}$ & $\begin{array}{l}-0.324 * * * \\
(0.015)\end{array}$ & $\begin{array}{l}-0.343^{* * *} \\
(0.020)\end{array}$ & $\begin{array}{l}-0.352 * * * \\
(0.018)\end{array}$ \\
\hline \multicolumn{6}{|c|}{ Central \& West \& South states } \\
\hline Chhattisgar & $\begin{array}{l}-0.163 * * * \\
(0.028)\end{array}$ & $\begin{array}{l}-0.202 * * * \\
(0.015)\end{array}$ & $\begin{array}{l}-0.254 * * * \\
(0.019)\end{array}$ & $\begin{array}{l}-0.231 * * * \\
(0.028)\end{array}$ & $\begin{array}{l}-0.243 * * * \\
(0.051)\end{array}$ \\
\hline Madhya Pradesh & $\begin{array}{l}-0.218 * * * \\
(0.023)\end{array}$ & $\begin{array}{l}-0.209 * * * \\
(0.019)\end{array}$ & $\begin{array}{l}-0.227 * * * \\
(0.012)\end{array}$ & $\begin{array}{l}-0.262^{* * * *} \\
(0.018)\end{array}$ & $\begin{array}{l}-0.292^{* * *} \\
(0.028)\end{array}$ \\
\hline Gujrat & $\begin{array}{l}0.118 * * * \\
(0.022)\end{array}$ & $\begin{array}{l}0.124 * * * \\
(0.017)\end{array}$ & $\begin{array}{l}0.0822 * * * \\
(0.011)\end{array}$ & $\begin{array}{l}0.0212 * \\
(0.013)\end{array}$ & $\begin{array}{l}-0.0526^{* * * *} \\
(0.014)\end{array}$ \\
\hline Maharastra & $\begin{array}{l}-0.0118 \\
(0.015)\end{array}$ & $\begin{array}{l}-0.0174 \\
(0.013)\end{array}$ & $\begin{array}{l}-0.0281 * * \\
(0.012)\end{array}$ & $\begin{array}{l}-0.0335^{*} \\
(0.020)\end{array}$ & $\begin{array}{l}-0.0493 * * \\
(0.022)\end{array}$ \\
\hline
\end{tabular}


Table 2 continued

\begin{tabular}{clllll}
\hline Estimates of simultaneous quantile regression & & & \\
\hline Independent variable & $\mathrm{q} 10$ & $\mathrm{q} 25$ & $\mathrm{q} 50$ & $\mathrm{q} 75$ & $\mathrm{q} 90$ \\
\hline Karnataka & $-0.0671^{* * *}$ & $-0.0749^{* * *}$ & $-0.117^{* * *}$ & $-0.130^{* * *}$ & $-0.150^{* * *}$ \\
\multirow{2}{*}{ Kerala } & $(0.018)$ & $(0.015)$ & $(0.012)$ & $(0.014)$ & $(0.026)$ \\
& 0.0381 & $0.0830^{* * *}$ & $0.0664^{* * *}$ & $0.0711^{* * *}$ & $0.0981^{* * *}$ \\
Tamil Nadu & $(0.026)$ & $(0.019)$ & $(0.016)$ & $(0.018)$ & $(0.032)$ \\
& $-0.143^{* * *}$ & $-0.126^{* * *}$ & $-0.154^{* * *}$ & $-0.148^{* * *}$ & $-0.146^{* * *}$ \\
Constant & $(0.014)$ & $(0.017)$ & $(0.012)$ & $(0.011)$ & $(0.020)$ \\
& $5.726^{* * *}$ & $5.963^{* * *}$ & $6.181^{* * *}$ & $6.443^{* * *}$ & $6.807^{* * *}$ \\
Observations & $(0.069)$ & $(0.030)$ & $(0.038)$ & $(0.041)$ & $(0.094)$ \\
\hline
\end{tabular}

$* P<0.05, * * P<0.01, * * * P<0.001$

Standard errors are reported in parenthesis

The dependent variable is log per-capita consumption expenditure. The base category for the occupation is proportion of economically active individuals in the household who are self-employed; for marital status, unmarried; for general/technical education, no general/technical education, and for state level regional dummies, the excluded state is Andhra Pradesh

expenditure. A $1 \%$ increase in the proportion of elderly people increases the per-capita consumption by $18 \%$ at the lowest quantile and $38 \%$ at the highest quantile. The proportion of females has an insignificant effect in the lower two quantiles but a significant positive effect at higher quantiles. Thus, at median, a $1 \%$ increase in the proportion of females increases the per-capita consumption by $4.4 \%$, and at $q(.9)$, by $9 \%$. The coefficients of the household size variables show that the relationship between household size and welfare of the household is consistent with earlier studies that households of larger size have a lower per-capita consumption expenditure. However, the household size squared term is positive and increases across quantiles, indicating that households of larger size become worse off along the quantiles, but at decreasing rates. Thus, a convex relationship exists between household size and welfare, with households in the middle of the distribution showing the greatest negative effect of size on per-capita consumption. This could be the result of higher economies of scale at the tails of the income distribution.

The quantile plots in Fig. 2 allow visualization of the estimated effects of the occupation variables on the per-capita consumption expenditure at different quantiles of the distribution. The $X$-axis shows the quantiles and the $Y$-axis shows the magnitude of the effect of the independent variables on the dependent variable. The dotted line shows the effect of the independent variable on the dependent variable if a simple ordinary least squares (OLS) regression is estimated. The curve shows the estimated effect of the independent variables on the per-capita consumption expenditure across different quantiles. The grey region around the curve shows the $95 \%$ confidence interval for the estimated effects. As the quantile plots in Fig. 2 show, the effect of occupation on the per-capita expenditure is non-linear and varies across quantiles. As the first sub-plot shows, the effect of being employers on per-capita consumption expenditure increases across the quantiles, varying from 0.34 at the lowest quantile to 0.45 at the highest quantile. Although the effect is significantly positive throughout, the magnitude of the effect is much smaller at the lower quantiles than at the higher quantiles. The second sub-plot shows that being salaried employees has a positive effect on per-capita consumption expenditure. However, the effect has an inverted U shape across quantiles. Similar non-linear effects are seen in the case of laborers and unemployed-the effects, however, are negative in both cases. Thus, estimating the mean effect of occupations on per-capita consumption expenditure in a simple OLS framework would not have captured these nonlinear effects.

Individuals choose occupations by maximizing expected utility over lifetime. As one example, a 

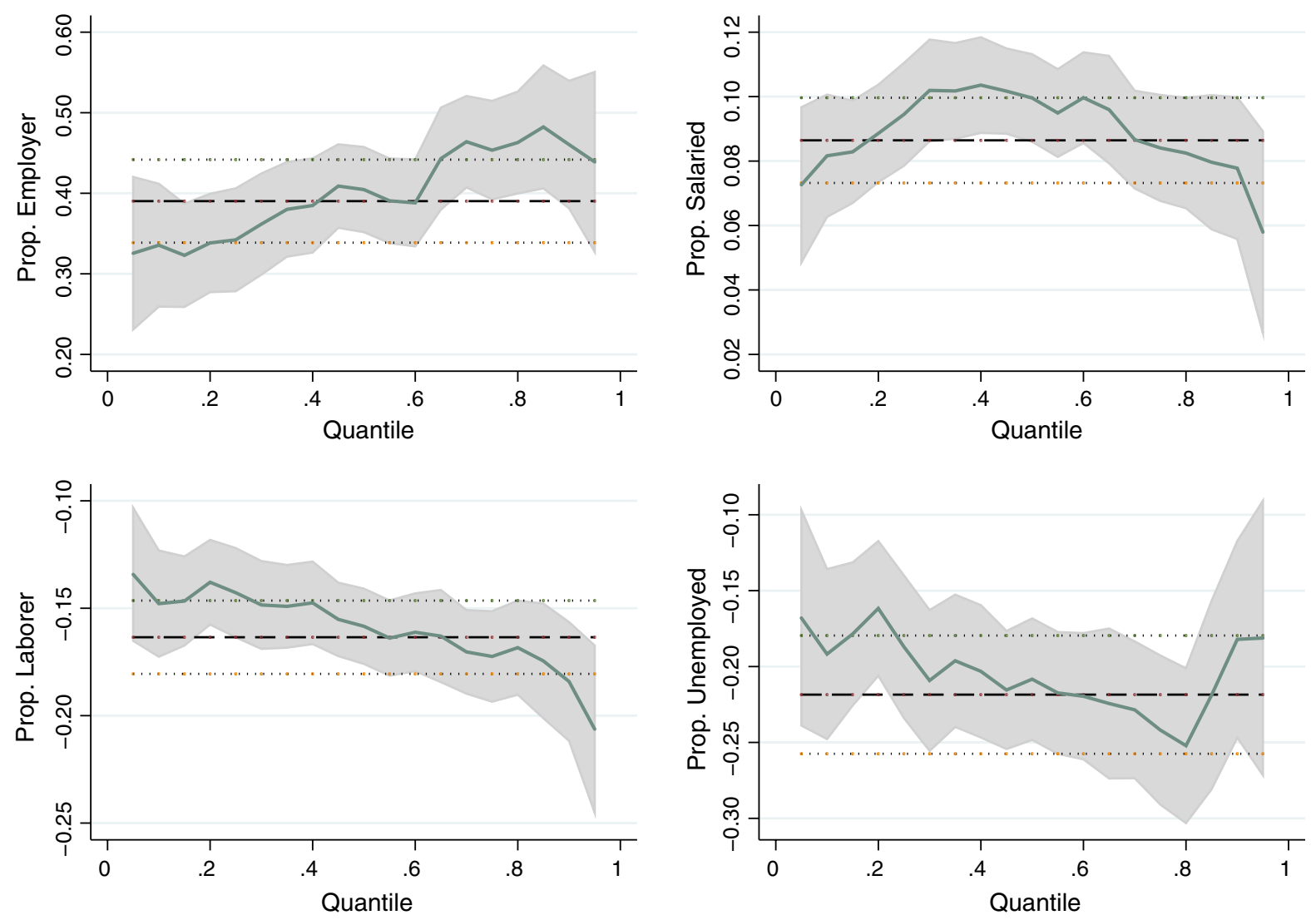

Fig. 2 Quantile plots. The graph shows the non-linear effects of the occupation variables, across different quantiles, on the per-capita consumption expenditure. The quantiles are on the

$X$-axis, and the estimated effects of occupations on the percapita consumption expenditure are on the $Y$-axis

higher degree of risk aversion and a preference for stability at the workplace may make individuals choose salaried employment, even if their income in the immediate future is relatively lower than that of the entrepreneurs. However, due to the cross-sectional nature of the database, this study is limited to examining the relative positioning of individuals in different occupational groups, across the welfare distribution. The results should be interpreted carefully given this caveat.

\subsubsection{Analysis restricted to household heads}

One of the main limitations of the analysis of the household level occupation data is the simultaneous determination of the occupation of the household members leading to potential endogeneity of the occupation variables. Thus, the occupation of members of the household may not be independent of the

\footnotetext{
$\overline{13 \text { A different }}$ source of endogeneity may arise as personal characteristics, such as age and educational background of the household members, may determine their occupational choice. However, the main aim of the paper is to examine if a welfare hierarchy of occupations is present across the welfare distribution, conditional on holding individual as well as household characteristics constant; the second issue is left for future research.
} 
entrepreneurship in particular, on household welfare. $^{14}$

The dataset on the unemployed is also dropped from the analysis as there are only 90 heads of household who are unemployed. Furthermore, as a check for robustness of the results in Table 2, the industry sector of the individuals in Table 3 is controlled as there may be sectoral differences in returns to self-employment. ${ }^{15}$ The base category for the occupation variables is 'salaried employee'. The estimation results are consistent with the estimations of the quantile regressions presented in Table 2. The results presented in Table 3 confirm the welfare hierarchy that the earlier regression suggested. Households headed by employers and salaried individuals have a higher per-capita consumption than households headed by self-employed individuals and casual laborers-after other factors that influence household welfare have been controlled. The magnitude of the coefficient of 'employer' suggests that households headed by entrepreneurs who employ others have the highest consumption levels. Relative to households headed by salaried employees, households headed by employers have consumption rates that $22.4 \%$ higher at the smallest quantile (q0), $25.8 \%$ higher at the median (q50), and $30.6 \%$ higher at the largest quantile (q90). Although the coefficient of salaried employees is positive, it is small, and salaried employees are only slightly better off than those who are self-employed. ${ }^{16}$ Households headed by self-employed individuals have consumption rates that are $4.9 \%$ lower at the smallest quantile $\mathrm{q}(10)$, and $6.3 \%$ lower at the median (q50). As suggested by the insignificant coefficient on the self-employed variable in the q90 column, households headed by selfemployed individuals and salaried employees have no significant difference in the consumption rates at the upper end of the distribution. The casual laborers are last in the hierarchy. Laborers have consumption

\footnotetext{
$\overline{14}$ An alternate strategy would be to instrument the occupation of the household members using the occupation of the household head. However, as household heads are in the sample and the occupation of their parents is not known, this is not viable.

15 As the dataset had unemployed people earlier, industry effects could not be controlled.

${ }^{16}$ Hamilton (2000) postulates that lower returns to selfemployment may be attributed to the individual's choice for freedom, leading them to select self-employment.
}

rates that are $20 \%-24.5 \%$ lower across the distribution. Thus, the results support the conjecture that relative returns to occupations differ across the welfare distribution and a comparison of returns at only the mean would not uncover these nonlinear effects.

Table 3 suggests that at lower quantiles, informal education has a significantly positive effect on the per-capita consumption. The returns to primary school education increase along the quantiles. It is seen that at the lowest quantile $[\mathrm{q}(.1)]$, primary schooling increases the per-capita consumption of the household by $14 \%$. The coefficient, however, is higher at the highest quantile, q(.9), where it raises the per-capita consumption of household by $19 \%$. A similar effect is observed for other education variables. If the household head has a high school education, per-capita consumption expenditure increases by $23 \%$ at the lowest quantile and $36 \%$ at the highest quantile. Similarly, if the household head has a university education, the per-capita consumption of the household increases by $41 \%$ at the lowest quantile and by $73 \%$ at the highest quantile. Thus, education has a positive effect on the per-capita consumption and increases as individuals move from the lower to higher quantiles. The returns to technical degree/diploma are also positive and increase as individuals shift from the lower to the higher quantiles. ${ }^{17}$ The estimates of the control variables are in accordance with the hypotheses and are consistent with the estimation in Table 2.

\subsubsection{Entrepreneurship and poverty}

Per-capita consumption of individuals is predicted after estimating the quantile regression at different quantiles. ${ }^{18}$ The cumulative distribution plots of occupation-wise predicted values are shown in Fig. 3. The per-capita expenditure is on the $X$-axis and the cumulative probability is on the $Y$-axis. At any point $x$ on the $X$-axis, the cumulative distribution function of a particular occupational category would give the proportion of individuals in that occupational

\footnotetext{
17 As there are very few individuals with technical degrees or diplomas, we merge these into one variable.

18 The log-inverse transformation of the predicted values gives the value of the normalized per-capita consumption expenditure. These transformed values are used in the poverty analysis.
} 
Table 3 Household heads, occupation, and consumption

\begin{tabular}{|c|c|c|c|c|c|}
\hline \multicolumn{6}{|c|}{ Estimates of simultaneous quantile regression } \\
\hline Independent variable & $\mathrm{q} 10$ & $\mathrm{q} 25$ & $\mathrm{q} 50$ & $\mathrm{q} 75$ & q90 \\
\hline \multicolumn{6}{|l|}{ Occupation } \\
\hline Employer & $\begin{array}{l}0.224 * * * \\
(0.058)\end{array}$ & $\begin{array}{l}0.226 * * * \\
(0.044)\end{array}$ & $\begin{array}{l}0.258 * * * \\
(0.037)\end{array}$ & $\begin{array}{l}0.252^{* * * *} \\
(0.077)\end{array}$ & $\begin{array}{l}0.306 * * * \\
(0.069)\end{array}$ \\
\hline Self-employed & $\begin{array}{l}-0.0491 * * * \\
(0.013)\end{array}$ & $\begin{array}{l}-0.0579 * * * \\
(0.012)\end{array}$ & $\begin{array}{l}-0.0631 * * * \\
(0.012)\end{array}$ & $\begin{array}{l}-0.0564 * * * \\
(0.012)\end{array}$ & $\begin{array}{l}-0.0225 \\
(0.019)\end{array}$ \\
\hline Laborer & $\begin{array}{l}-0.228 * * * \\
(0.016)\end{array}$ & $\begin{array}{l}-0.229 * * * \\
(0.017)\end{array}$ & $\begin{array}{l}-0.246 * * * \\
(0.012)\end{array}$ & $\begin{array}{l}-0.225 * * * \\
(0.019)\end{array}$ & $\begin{array}{l}-0.203 * * * \\
(0.018)\end{array}$ \\
\hline \multicolumn{6}{|l|}{ Personal characteristics } \\
\hline Age & $\begin{array}{l}0.0340 * * * \\
(0.0047)\end{array}$ & $\begin{array}{l}0.0324 * * * \\
(0.0039)\end{array}$ & $\begin{array}{l}0.0395 * * * \\
(0.0039)\end{array}$ & $\begin{array}{l}0.0405 * * * \\
(0.0043)\end{array}$ & $\begin{array}{l}0.0282 * * * \\
(0.0066)\end{array}$ \\
\hline Age square & $\begin{array}{l}-0.0371 * * * \\
(0.0061)\end{array}$ & $\begin{array}{l}-0.0329 * * * \\
(0.0050)\end{array}$ & $\begin{array}{l}-0.0409 * * * \\
(0.0048)\end{array}$ & $\begin{array}{l}-0.0399 * * * \\
(0.0051)\end{array}$ & $\begin{array}{l}-0.0240 * * * \\
(0.0083)\end{array}$ \\
\hline Female & $\begin{array}{l}-0.0144 \\
(0.035)\end{array}$ & $\begin{array}{l}-0.0296 \\
(0.031)\end{array}$ & $\begin{array}{l}-0.0653 \\
(0.043)\end{array}$ & $\begin{array}{l}0.0125 \\
(0.041)\end{array}$ & $\begin{array}{l}0.0811 \\
(0.060)\end{array}$ \\
\hline Married & $\begin{array}{l}-0.0301 \\
(0.037)\end{array}$ & $\begin{array}{l}-0.0312 \\
(0.021)\end{array}$ & $\begin{array}{l}-0.0321 \\
(0.029)\end{array}$ & $\begin{array}{l}-0.0658 * * * \\
(0.022)\end{array}$ & $\begin{array}{l}-0.0435 \\
(0.053)\end{array}$ \\
\hline Divorce/Widow & $\begin{array}{l}-0.212 * * * \\
(0.037)\end{array}$ & $\begin{array}{l}-0.233 * * * \\
(0.034)\end{array}$ & $\begin{array}{l}-0.176^{* * * *} \\
(0.042)\end{array}$ & $\begin{array}{l}-0.220 * * * \\
(0.034)\end{array}$ & $\begin{array}{l}-0.184^{* *} \\
(0.075)\end{array}$ \\
\hline \multicolumn{6}{|l|}{ General education } \\
\hline Informal education & $\begin{array}{l}0.0479 * \\
(0.027)\end{array}$ & $\begin{array}{l}0.0390 * * \\
(0.019)\end{array}$ & $\begin{array}{l}0.0219 \\
(0.025)\end{array}$ & $\begin{array}{l}0.0339 * \\
(0.018)\end{array}$ & $\begin{array}{l}0.0233 \\
(0.024)\end{array}$ \\
\hline Primary school & $\begin{array}{l}0.142 * * * \\
(0.018)\end{array}$ & $\begin{array}{l}0.146 * * * \\
(0.013)\end{array}$ & $\begin{array}{l}0.137 * * * \\
(0.018)\end{array}$ & $\begin{array}{l}0.172 * * * \\
(0.018)\end{array}$ & $\begin{array}{l}0.191 * * * \\
(0.016)\end{array}$ \\
\hline High school & $\begin{array}{l}0.235 * * * \\
(0.017)\end{array}$ & $\begin{array}{l}0.268 * * * \\
(0.014)\end{array}$ & $\begin{array}{l}0.292 * * * \\
(0.016)\end{array}$ & $\begin{array}{l}0.341 * * * \\
(0.015)\end{array}$ & $\begin{array}{l}0.361 * * * \\
(0.017)\end{array}$ \\
\hline University education & $\begin{array}{l}0.413 * * * \\
(0.025)\end{array}$ & $\begin{array}{l}0.483 * * * \\
(0.015)\end{array}$ & $\begin{array}{l}0.559 * * * \\
(0.019)\end{array}$ & $\begin{array}{l}0.640 * * * \\
(0.023)\end{array}$ & $\begin{array}{l}0.732 * * * \\
(0.022)\end{array}$ \\
\hline Technical Degree or Diploma & $\begin{array}{l}0.170 * * * \\
(0.021)\end{array}$ & $\begin{array}{l}0.180 * * * \\
(0.015)\end{array}$ & $\begin{array}{l}0.169 * * * \\
(0.016)\end{array}$ & $\begin{array}{l}0.191 * * * \\
(0.017)\end{array}$ & $\begin{array}{l}0.235^{* * * *} \\
(0.024)\end{array}$ \\
\hline Demographics & Yes & & & & \\
\hline Household characteristics & Yes & & & & \\
\hline Region controls & Yes & & & & \\
\hline Sector controls & Yes & & & & \\
\hline Constant & $\begin{array}{l}5.773 * * * \\
(0.085)\end{array}$ & $\begin{array}{l}6.081 * * * \\
(0.071)\end{array}$ & $\begin{array}{l}6.237 * * * \\
(0.072)\end{array}$ & $\begin{array}{l}6.478 * * * \\
(0.068)\end{array}$ & $\begin{array}{l}6.923 * * * \\
(0.12)\end{array}$ \\
\hline Observations & 13,692 & 13,692 & 13,692 & 13,692 & 13,692 \\
\hline
\end{tabular}

$* P<0.05, * * P<0.01, * * * P<0.001$

Standard errors are reported in parentheses

The dependent variable is $\log$ per-capita consumption expenditure. The base category for occupation is the salaried employee; for marital status, unmarried; for general/technical education, no general/technical education. A full set of state level regional dummies is also included in the regression, with the excluded state being Andhra Pradesh 

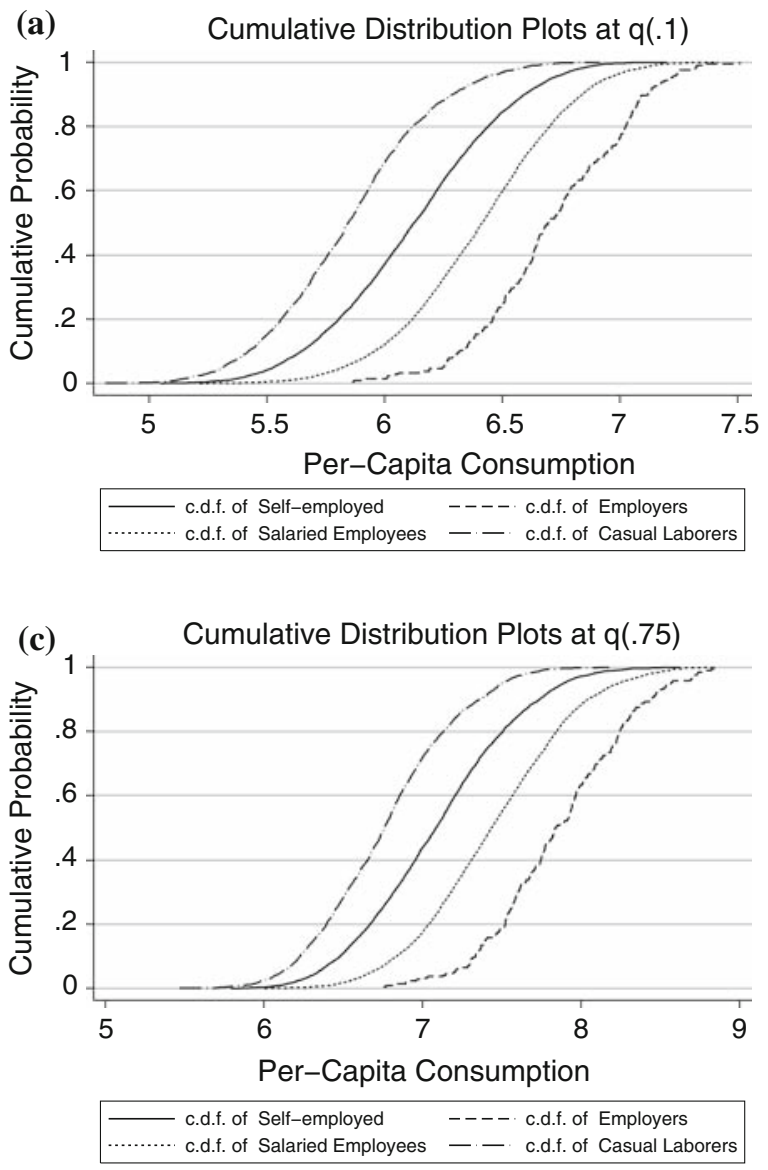

Fig. 3 Occupation and poverty plots

category whose consumption is below $x$. It can be seen that in all the sub-plots of Fig. 3, at any given point $x$ on the $X$-axis, the proportion of employers whose per-capita consumption expenditure is below $x$ is lower than the proportion of salaried employees whose per-capita expenditure is below $x$. An analogous argument implies that the proportion of salaried employees whose per-capita consumption expenditure is below $x$ is lower than the proportion of selfemployed individuals or casual laborers whose percapita consumption expenditure is below $x$. The plots show that the cumulative distribution function of employers stochastically dominates the distributions of other occupational categories. Since $x$ is any point on the $X$-axis, the figure does away with the necessity of having a fixed poverty line to examine the relative poverty in different occupational groups.

A simple mathematical formulation of the above arguments is as follows. Let the cumulative
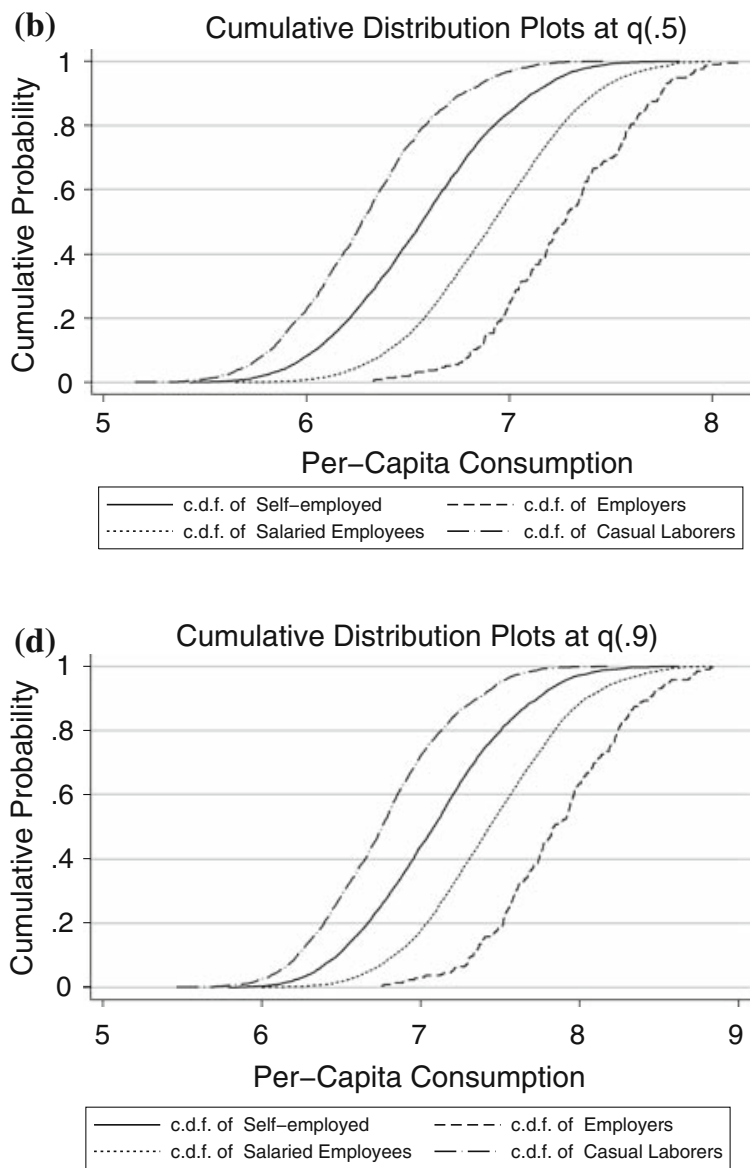

distribution functions of the four occupational categories be given by functions $F_{\text {emp }}$ for employers, $F_{\text {sal }}$ for salaried employees, $F_{\text {self }}$ for self-employed individuals, and $F_{\text {lab }}$ for laborers. At any given point $x$ on the $X$-axis, the plots show that that $F_{\text {emp }}(x)<$ $F_{\text {sal }}(x)<F_{\text {self }}(x)<F_{\text {lab }}(x)$. Thus, the employers group would have the least proportion of people under the poverty line, if the poverty line is at $x$.

The plot clarifies the status of the self-employed; they appear to be sandwiched between the salaried employees and the casual laborers. A direct implication of this observation is that, conditional on other characteristics, individuals in the informal sector, primarily comprising the self-employed and the casual laborers, have lower returns to their occupations. Furthermore, if the dataset is split into formal and informal sectors, with laborers and self-employed in the informal sector and salaried employees and employers in the formal sector, the plots suggest that 
in both sectors, entrepreneurship in the form of employers in the formal sector and self-employed in the informal sector entails higher relative consumption.

I also analyzed occupational choice as a determinant of poverty of households using a probit model. A widely used relative measure of poverty, given by half of the median per-capita consumption of all the households, was considered as the poverty line. ${ }^{19}$ The results suggest that while households headed by employers, self-employed, and salaried employees are less likely to be under the poverty line, households headed by casual laborers are most likely to be under the poverty line, after controlling many characteristics that are likely to influence their poverty status. $^{20}$

\section{Conclusion}

This paper makes important contributions to the literature on the economics of entrepreneurship. I have extensively examined the welfare consequences of entrepreneurship in a developing country, an area of study that has received little attention to date.

Using simultaneous quantile regressions, I have found that employers, those entrepreneurs who also hire others, have the highest returns in terms of consumption, while the self-employed, those entrepreneurs who work for themselves, have slightly lower returns than the salaried employees. The results demonstrate that the relative returns to occupations differ at different points of the welfare distribution. Thus, empirical studies that consider only mean returns for broad occupational categories miss important variations in the data. For instance, although the consumption rates of self-employed household heads is significantly lower at many points of the distribution relative to the salaried household heads, there is no significant difference at the highest quantile. A comparison of returns at the mean alone would not have uncovered these nonlinear effects. Selfemployed are more likely to escape poverty, as are salaried employees and entrepreneurs who are

\footnotetext{
${ }^{19}$ Using an alternate poverty line based on the number of adults has not significantly altered the main inferences.

${ }^{20}$ For brevity these results are not reported here but are available from the author.
}

employers, when compared to casual laborers. The results are robust to alternate empirical specifications.

Open Access This article is distributed under the terms of the Creative Commons Attribution Noncommercial License which permits any noncommercial use, distribution, and reproduction in any medium, provided the original author(s) and source are credited.

\section{References}

Banerjee, A. V., \& Neuman, A. F. (1993). Occupational choice and the process of economic development. Journal of Political Economy, 101, 274-298.

Benito, A. (2006). Does job insecurity affect household consumption? Oxford Economic Papers, 58(1), 157-181.

Blanchflower, D. G., \& Oswald, A. (1998) What makes an entrepreneur. Journal of Labor Economics, 16(1), 26-60.

Blau, D. M. (1987). A time series analysis of self-employment in United States. Journal of Political Economy, 95(3), 445-467.

Boháček, R. (2006). Financial constraints and entrepreneurial investment. Journal of Monetary Economics, 53(8), 21952212.

Browning, M. (1992) Children and household economic behavior. Journal of Economic Literature, 30(3), 14341475.

Browning, M., \& Lusardi, A. (1996). Household saving: Micro theories and micro facts. Journal of Economic Literature, 34(4), 1797-1855.

Browning, M., \& Crossley, T. F. (2001). The life-cycle model of consumption and saving. Journal of Economic Perspectives, Summer 15(3), 3-22.

Cunningham, W. V., \& Maloney, W. F. (2001). Heterogeneity in the Mexican micro-enterprise sector. Economic Development and Cultural Change, 50, 131-156.

Dabla-Norris, E., Gradstein, M., \& Inchauste, G. (2008). What causes firms to hide output? The determinants of informality. Journal of Development Economics, 85, 1-27.

Deaton, A. S., \& Paxson, C. H. (1995). Measuring poverty among the elderly. NBER Working Paper 5296. Cambridge: National Bureau of Economic Research

Deaton, A. S., \& Muellbauer, J. (1980). Economics and consumer behavior. Cambridge: Cambridge University Press.

Deaton, A. S., \& Muellbauer, J. (1986). On measuring child costs: With applications to poor countries. The Journal of Political Economy, 94(4), 720-744.

Dreze, J., \& Srinivasan, P. V. (1997). Widowhood and poverty in rural India: Some inferences from household survey data. Journal of Development Economics, 54(2), 217-234.

Evans, D. S., \& Leighton, L. S. (1989). Some empirical aspects of entrepreneurship. American Economic Review, 79(3), 519-535.

Fields, G. S. (2005). A guide to multisector labor market models. Social protection discussion paper series No. 0505. Washington D.C.: World Bank.

Gindling, T. H. (1991). Labor market segmentation and the determination of wages in the public, private-formal and 
informal sectors in San-Jose, Costa-Rica. Economic Development and Cultural Change, 39, 585-605.

Glewwe, P. (1991). Investigating the determinants of household welfare in Côte d'Ivoire. Journal of Development Economics, 35(2), 307-337.

Günther, I., \& Launov, A. (2006). Competitive and segmented informal labor markets. IZA discussion papers 2349 . Bonn: Institute for the Study of Labor (IZA).

Hamilton, B. H. (2000). Does entrepreneurship pay? An empirical analysis of the returns of self-employment. Journal of Political Economy, 108(3), 604-631.

Harris, J. R., \& Todaro, M. P. (1970). Migration, unemployment and development: A two sector analysis. American Economic Review, 60(1), 126-142.

Jenkins, S. P. (2000) Modelling household income dynamics. Journal of Population Economics, 13(4), 529-567.

Kihlstrom, R. E., \& Laffont, J. J. (1979). A general equilibrium entrepreneurial theory of firm formation based on risk aversion. Journal of Political Economy, 87, 719-748.

Koenker, R., \& Hallock, H. F. (2001). Quantile regression: An introduction. Journal of Economic Perspectives,15(4), 143-156.

Lanjouw, P., \& Ravallion, M. (1995). Poverty and household size. Economic Journal, 105(433), 1415-1434.

Lazear, E. P., \& Michael, R. T. (1980). Family size and the distribution of real per capita income. American Economic Review, 70(1), 91-107.

Lucas, R. (1978). On the size distribution of business firms. Bell Journal of Economics, 9, 508-523.

Magnac, T. (1991). Segmented or competitive labor markets. Econometrica, 59, 165-187.

Maloney, W. F. (2004). Informality revisited. World Development, 32(7), 1159-1178.
Miles, D. (1997). A household level study of the determinants of incomes and consumption. Economic Journal, 107(440), 1-25.

Muellbauer, J. (1974). Household composition, engel curves and welfare comparisons between households. European Economic Review, 5, 103-122.

Nelson, J. A. (1988). Household economies of scale in consumption: Theory and evidence. Econometrica, 56(6), 1301-1314.

Parker, S. C. (1996). A time series model of self-employment under uncertainty. Economica, 63, 469-475.

Parker, S. C. (2004). The economics of self-employment and entrepreneurship. Cambridge: Cambridge University Press

Pradhan, M., \& Ravallion, M. (2000). Measuring poverty using qualitative perceptions of consumption adequacy. The Review of Economics and Statistics, 82(3), 462-471.

Pratap, S., \& Quintin, E. (2006). Are labor markets segmented in developing countries? A semiparametric approach European Economic Review, 50, 1817-1841.

Ranis, G., \& Fei, J. (1961). A theory of economic development. American Economic Review, 51, 533-565.

Rees, H., \& Shaw, A. (1986). An empirical analysis of selfemployment in UK. Journal of Applied Econometrics, 1, 95-108.

Rosen, S. (1981). The economics of superstars. American Economic Review, 71(5), 845-858.

Wodon, Q. T. (2000). Microdeterminants of consumption, poverty, growth, and inequality in Bangladesh. Applied Economics, 32(10), 1337-1352. 